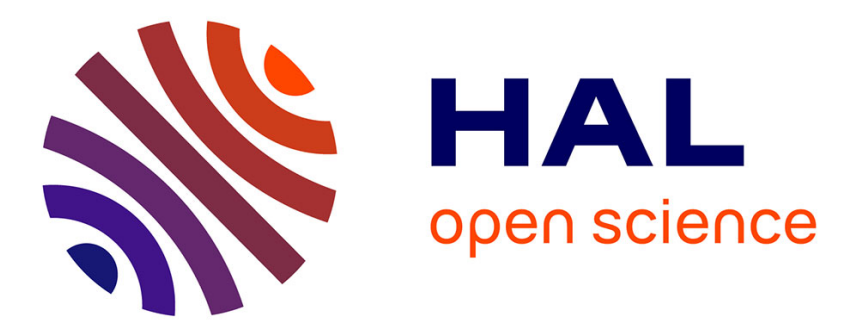

\title{
An aqueous miscible organic (AMO) process for layered double hydroxides (LDHs) for the enhanced properties of polypropylene/LDH composites
}

Qian Zhang, Yixuan Guo, Fabrice Leroux, Pinggui Tang, Dianqing Li, Liren Wang, Yongjun Feng

\section{To cite this version:}

Qian Zhang, Yixuan Guo, Fabrice Leroux, Pinggui Tang, Dianqing Li, et al.. An aqueous miscible organic (AMO) process for layered double hydroxides (LDHs) for the enhanced properties of polypropylene/LDH composites. New Journal of Chemistry, 2020, 44 (24), pp.10119-10126. 10.1039/C9NJ06444C . hal-02992455

\section{HAL Id: hal-02992455 https://hal.science/hal-02992455}

Submitted on 20 Nov 2020

HAL is a multi-disciplinary open access archive for the deposit and dissemination of scientific research documents, whether they are published or not. The documents may come from teaching and research institutions in France or abroad, or from public or private research centers.
L'archive ouverte pluridisciplinaire HAL, est destinée au dépôt et à la diffusion de documents scientifiques de niveau recherche, publiés ou non, émanant des établissements d'enseignement et de recherche français ou étrangers, des laboratoires publics ou privés. 


\title{
An aqueous miscible organic (AMO) process for layered double hydroxides (LDHs) for the enhanced properties of polypropylene/LDH composites
}

\begin{abstract}
Qian Zhang, ${ }^{\text {ta }}$ Yixuan Guo, ${ }^{\text {ta }}$ Fabrice Leroux, ${ }^{\mathrm{b}}$ Pinggui Tang, ${ }^{\mathrm{a}}$ Dianqing Li, ${ }^{\mathrm{a}}$ Liren Wang*c and Yongjun Feng*a,c
Enhancing the compatbility between inorgnic filler and polymer to obtain functionalized composite has always been a key issue. An aqueous miscible organic (AMO) method is here successfully adapted for the conventional layered double hydroxides (LDH) fillers. Here, a series of D-LDH hybrid materials, intercalated by an organic antioxidant DBHP (D, 3-(3,5-ditertbutyl-4-hydroxyphenyl) propionate), have been organically surface-modified using acetone as the solvent through the AMO method. To understand the effect of acetone exposure on the structure of AMO D-LDH samples, XRD, FT-IR, TG-DTA and radical-scavenging activity were thoroughly characterized. The results indicated that the AMO method does not affect the layer structure of D-LDH but causes the co-intercalation of $\mathrm{D}^{-}$with $\mathrm{CO}_{3}{ }^{2-}$ anions, which change the amount of DBHP in the interlayer and then influence the crystallinity, thermal stability and the radical-scavenging activity of DLDH. Subsequently, the series of AMO D-LDH was used as an anti-aging nanofiller for polypropylene (PP). The thermal stability, thermalaging properties and migration resistance of the resulting PP/D-LDH (h) composites were investigated systematically. The results showed that the AMO modification of D-LDH affects the content of DBHP and the interaction between LDH filler and PP chains, since an optimized modification time and content of DBHP are found to enhance the anti-aging performance of PP, as well as to limit the migration of DBHP molecule out of PP films.
\end{abstract}

\section{Introduction}

Inorganic nanofillers have been widely developed to improve the diverse properties of polymer nanocomposites regarding their mechanical strength, thermal stability, flame retardation and so on. ${ }^{1-}$ ${ }^{3}$ Among the investigated inorganic fillers, layered double hydroxides $(\mathrm{LDHs})\left[\mathrm{M}^{2+}{ }_{1-x} \mathrm{M}^{3+}{ }_{x}(\mathrm{OH})_{2}\right]\left(\mathrm{A}^{\mathrm{n}-}\right.$ $\mathrm{x} / \mathrm{n}) \cdot \mathrm{mH}_{2} \mathrm{O}$ have been attracting considerable attention due to the versatile controllable regulation of their chemical compositions involving both the interlayer gallery anions $\left(A^{n-}\right)$ and the inorganic layers $\left(\mathrm{M}^{2+}, \mathrm{M}^{3+}\right),{ }^{4,5}$ which result in remarkably tunable properties for wide applications, e.g., polymer, catalysis, environment, electrochemistry as well as delivery-vessels in biology. ${ }^{6-10}$

Besides chemical composition, structure and morphology, dispersion degree of LDH nanoparticles in polymer plays the key role in enhanced performances of the composites. ${ }^{11}$

However, high surface charge density, high surface energy and hydrophilic nature generally cause heavy aggregation of LDH nanoparticles and then obviously reduce the corresponding dispersion degree during the fabrication process of polymer/LDH composites. ${ }^{12}$ Few successful strategies have been provided and investigated to overcome this crucial issue. Recently, a simple method, called aqueous miscible organic (AMO) method, ${ }^{13}$ has been developed to improve the compatibility of LDHs with polymer and significantly enhance the dispersion degree in polymer, and then optimize various performances of polymer. ${ }^{14}$ For example, Gao et al. ${ }^{15}$ used the AMO method to fabricate polypropylene (PP)/LDH-OCNT (oxidized carbon nanotube) nanocomposites, and shown the stronger synergistic effect among LDH, OCNT, and PP with enhanced performance in terms of flame retardancy, thermal stability, and mechanical properties. All these results prompt us to apply the AMO process for an intercalated anti-aging agent for $\mathrm{PP}$, and to carefully scrutinize the effect of the exposure in acetone of the hybrid LDH filler.

In our previous work, a series of antioxidants $(\mathrm{AO})$ and light stabilizers with intercalated structure have been designed and exhibited excellent improved anti-aging performances of the polymer/AO$\mathrm{LDH}$ composites, related to the functional guests before intercalation into the $\mathrm{LDHs}{ }^{16,17}$ For example, 3-(3,5-ditertbutyl-4-hydroxyphenyl) propionic acid (DBHP, signed as D, one-fourth of Irganox 1010, which is the most used antioxidant in industry) was assembled into the interlayer of $\mathrm{Mg}_{2} \mathrm{Al}-\mathrm{LDH}$ to generate intercalated D-LDH. The prepared D-LDH-type filler showed an enhanced long-term thermal stability in comparison to $\mathrm{PP}, \mathrm{CO}_{3}-\mathrm{LDH} / \mathrm{PP}$ and $\mathrm{D} / \mathrm{PP}$, thus being a promising antioxidant for PP. In addition, D-LDH/PP composites were found to maintain the DBHP molecule inside the composites 
with an improved anti-migration behavior. ${ }^{18}$ Having in mind the additional advantages of AMO method, we designed and fabricated a series of AMO D-LDH (h) with different acetone modification times $(0,1,3,6,9,12 \mathrm{~h})$. The AMO D-LDH (h) were used as the anti-aging nanofiller, followed by mixing with PP to prepare the PP/D-LDH (h) nanocomposites. The AMO D-LDH (h) were thoroughly characterized by XRD, FT-IR, TG-DTA, in order to understand the effect of AMO modification time on the structure of D-LDHs. Furthermore, the influence of the addition of AMO D-LDH (h) on the thermal stability, thermo-oxidative degradation behavior and possible migration property of PP was systematically evaluated. Scheme 1 describes the synthesis process of AMO D-LDH (h) and PP/D-LDH (h) composites.
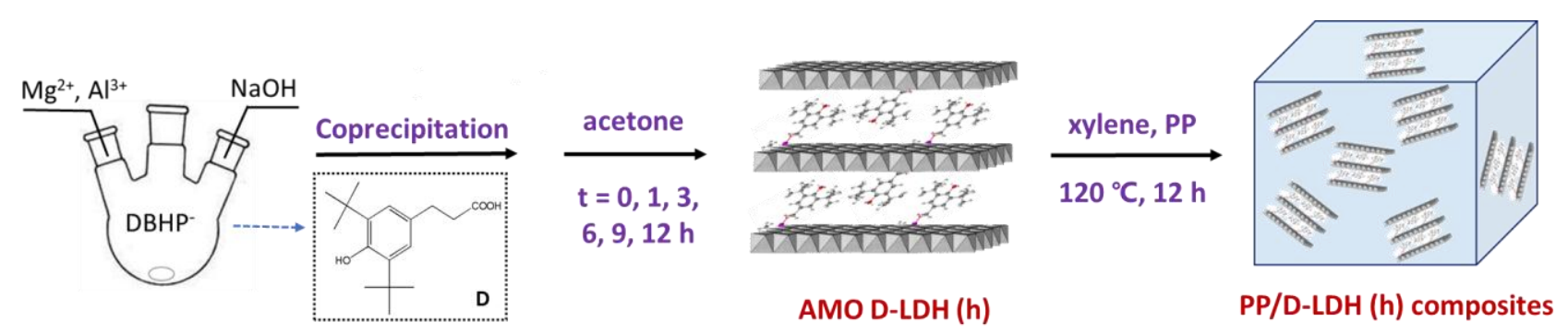

Scheme 1. Synthesis process of AMO D-LDH (h) and PP/D-LDH (h) composites.

Results and discussion

A series of AMO D-LDH (h) antioxidants were prepared by dispersing D-LDH wet cake in acetone solvent under vigorous stirring at room temperature from $t=0$ to $12 \mathrm{~h}$. Also, the corresponding PP/D$\mathrm{LDH}(\mathrm{h})$ nanocomposites were fabricated by adding a certain amount of D-LDH(h) powder into xylene solution of PP in a solvent mixing route. The anti-aging performance and the structure-performance relationship of PP/D-LDH (h) nanocomposites were carefully investigated and discussed.

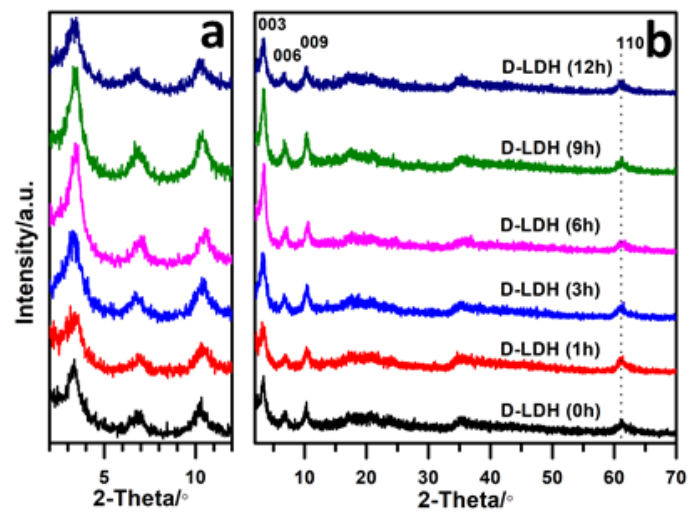

Fig. 1 (a) Enlarged 20, low domain and (b) full domain XRD patterns of D-LDH (h).

\section{Structure of AMO D-LDH (h)}

Fig. 1 displays the powder X-ray diffraction (XRD) patterns of the series D-LDH (h) ( $t=0$ to $12 \mathrm{~h}$ ), where $h$ is the contact time between the acetone and the D-LDH. One observes four characteristic reflections of $\mathrm{Mg}_{2} \mathrm{Al}-\mathrm{LDH}$ as indexed to the (003), (006), (009) and (110) Bragg reflections, suggesting the formation of layered structure and an intra-layer structural ordering, respectively. ${ }^{19}$ As for all the D-LDH (h) samples, the (003) diffraction reflection peak locates about $3.4^{\circ} / 2 \theta$, corresponding to a basal spacing distance of $\sim 2.6 \mathrm{~nm}$. Besides, the chemical composition of D-LDH (h) are obtained according to the TG results, as shown in Table 1. In the process of AMO treatment, an uptake of $\mathrm{CO}_{2}$ occurred and caused a strong contamination that induces the co-intercalation of $\mathrm{D}^{-}$and $\mathrm{CO}_{3}{ }^{2-}$ in $\mathrm{D}$ - 
LDH (h). This may be explained by the fact that the particle surface after AMO treatment is more sensitive to contamination with carbonate anions. ${ }^{14}$ One observes also some pillaring effect due to the cumbersome DBHP molecule, leading to some minor variations in the basal spacing values. Besides, D-LDH (h) exhibits slightly different states of crystallinity. ${ }^{20}$ The Bragg reflections of some DLDHs are broader with a lower intensity, which is typical for a decrease in the structural coherence length, and that is interpreted here for the hybrid LDH assembly by a limited number of stacked layers too small to be considered as long-range order. ${ }^{21}$ Moreover, no diffraction peaks for carbonate phase $\left(\mathrm{Mg}_{2} \mathrm{Al}-\mathrm{CO}_{3}-\mathrm{LDH}\right.$ presents a basal spacing value of $\left.0.76 \mathrm{~nm}\right)$ are observed, because the $\mathrm{D}^{-}$anion is of larger size than $\mathrm{CO}_{3}{ }^{2-}$ anion. That is to say, the $\mathrm{AMO}$ method does not affect the layer structure of D-LDH but results in the co-intercalation of $\mathrm{D}^{-}$and $\mathrm{CO}_{3}{ }^{2-}$.

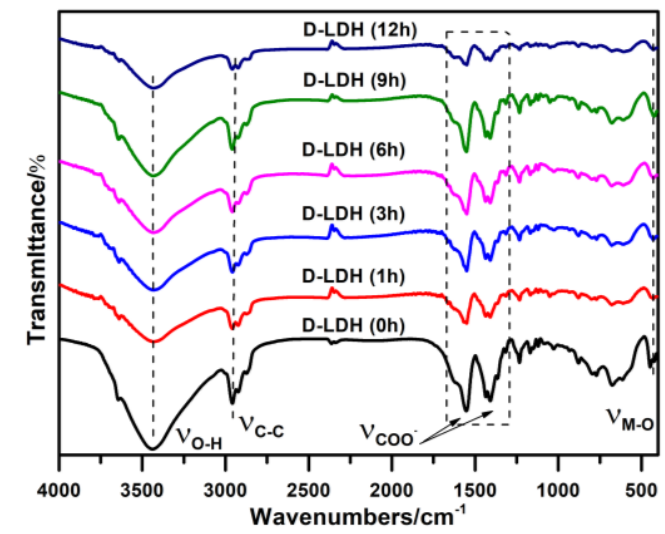

Fig. 2 FT-IR spectra of D-LDH (h).

Table 1 Chemical compositions and TG-DTA results of D-LDH (h).

\begin{tabular}{lllllll}
\hline Samples & Chemical composition & $\begin{array}{l}\text { Molecular } \\
\text { weight }(\mathrm{g} / \mathrm{mol})\end{array}$ & $\begin{array}{l}\text { DBHP } \\
\text { content }(\%)\end{array}$ & $\begin{array}{l}\mathrm{T}_{50 \%} \\
\left({ }^{\circ} \mathrm{C}\right)\end{array}$ & $\begin{array}{l}\text { DTA peak } \\
\left({ }^{\circ} \mathrm{C}\right)\end{array}$ & $\begin{array}{l}\text { Res. mass } \\
(\mathrm{wt} \%)\end{array}$ \\
\hline D-LDH (Oh) & $\mathrm{Mg}_{2} \mathrm{Al}(\mathrm{OH})_{6}\left(\mathrm{D}^{-}\right)_{0.94}\left(\mathrm{CO}_{3}{ }^{2-}\right)_{0.03} \cdot 4.45 \mathrm{H}_{2} \mathrm{O}$ & 519.28 & 50.14 & 305 & 333 & 26 \\
D-LDH (1h) & $\mathrm{Mg}_{2} \mathrm{Al}(\mathrm{OH})_{6}\left(\mathrm{D}^{-}\right)_{0.63}\left(\mathrm{CO}_{3}{ }^{2-}\right)_{0.18} \cdot 2.7 \mathrm{H}_{2} \mathrm{O}$ & 410.91 & 42.47 & 339 & 396 & 33 \\
$\mathrm{D}-\mathrm{LDH}(3 \mathrm{~h})$ & $\mathrm{Mg}_{2} \mathrm{Al}(\mathrm{OH})_{6}\left(\mathrm{D}^{-}\right)_{0.38}\left(\mathrm{CO}_{3}{ }^{2-}\right)_{0.31} \cdot 2.61 \mathrm{H}_{2} \mathrm{O}$ & 347.84 & 30.26 & 351 & 376 & 38 \\
D-LDH (6h) & $\mathrm{Mg}_{2} \mathrm{Al}(\mathrm{OH})_{6}\left(\mathrm{D}^{-}\right)_{0.94}\left(\mathrm{CO}_{3}{ }^{2-}\right)_{0.03} \cdot 3.72 \mathrm{H}_{2} \mathrm{O}$ & 506.14 & 51.44 & 323 & 397 & 27 \\
D-LDH (9h) & $\mathrm{Mg}_{2} \mathrm{Al}(\mathrm{OH})_{6}\left(\mathrm{D}^{-}\right)_{0.5}\left(\mathrm{CO}_{3}{ }^{2-}\right)_{0.25} \cdot 2.53 \mathrm{H}_{2} \mathrm{O}$ & 376.04 & 36.83 & 347 & 370 & 36 \\
D-LDH (12h) & $\mathrm{Mg}_{2} \mathrm{Al}(\mathrm{OH})_{6}\left(\mathrm{D}^{-}\right)_{0.42}\left(\mathrm{CO}_{3}{ }^{2-}\right)_{0.22} \cdot 2.69 \mathrm{H}_{2} \mathrm{O}$ & 357.16 & 32.39 & 363 & 354 & 37 \\
\hline
\end{tabular}

Fig. 2 depicts the FT-IR spectra of the D-LDH (h) with the characteristic IR absorption bands of $\mathrm{Mg}_{2} \mathrm{Al}-$ LDH. For example, the broad band at ca. $3435 \mathrm{~cm}^{-1}$ is assigned to the $\mathrm{O}-\mathrm{H}$ stretching vibration of water molecules and the hydroxyl in the brucite-like layer. The band at $425 \mathrm{~cm}^{-1}$ is attributed to the lattice vibration ( $\mathrm{M}-\mathrm{O}$ and $\mathrm{O}-\mathrm{M}-\mathrm{O}$ ) from $\mathrm{LDH}$ sheets, thus proving the formation of $\mathrm{Mg}_{2} \mathrm{Al}-\mathrm{LDH}$ edgesharing $\mathrm{OH}$ structure. Furthermore, the typical stretching vibration bands of DBHP species also occur, such as its carbonyl group and its alkyl group as marked in graph. Moreover, the splitting between the asymmetrical and symmetrical mode of carbonyl stretching vibrations $\left(\Delta \mathrm{v}\left(\mathrm{COO}^{-}\right)=\mathrm{v}_{\mathrm{as}}-\mathrm{v}_{\mathrm{s}}\right)$ is indicative of a coordination mode between the functional group of the carboxylate ion and the hydroxyl-based inner space of the LDH host.22 The vas and vs values remain at the same positions (1551 and $1408 \mathrm{~cm}-1$ ) along the series, and $\Delta v$ of about $140 \mathrm{~cm}-1$ corresponds to a coordination mode adopting a chelating character. Also, a weak absorption band of acetone is noticed at ca. 1620 cm-1 for all D-LDH (h). That is to say, some of acetone molecules are attached onto the LDH layer structure and there is however no change in the symmetry of the tethered DBHP group in the series. 

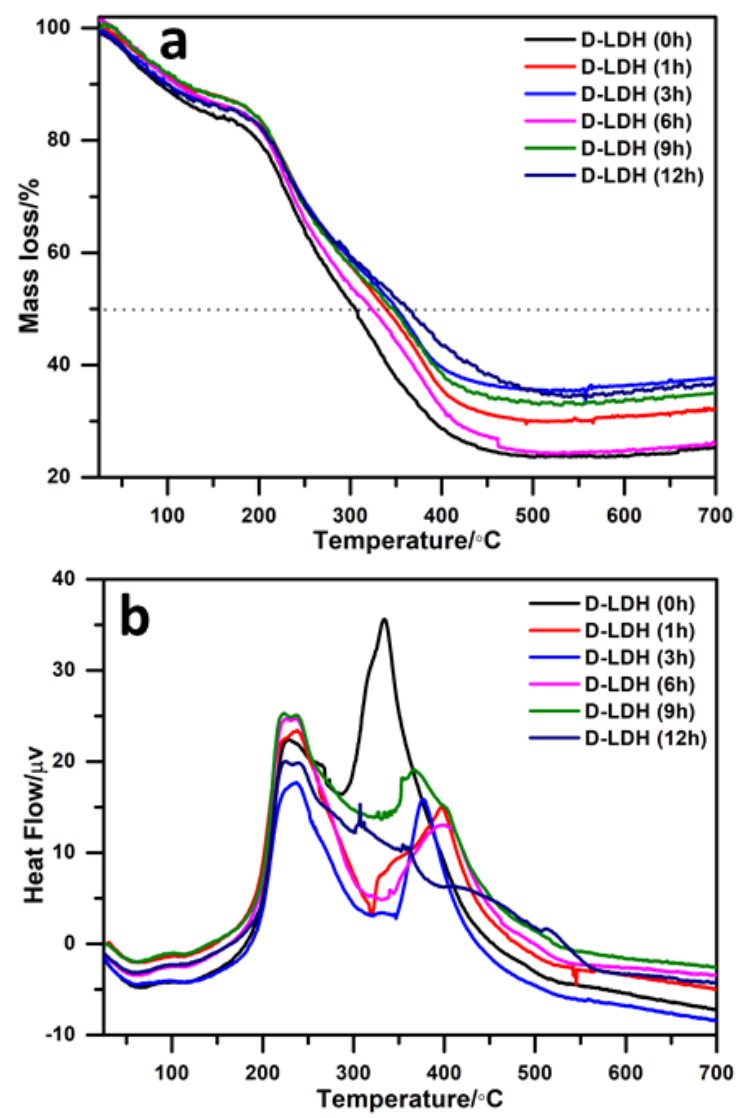

Fig. 3 (a) TG and (b) DTA curves of D-LDH (h).

Fig. 3 depicts the TG and DTA curves on the thermal stability of the D-LDH (h) samples and Table 1 summarizes the corresponding data. Here, three major mass loss steps are observed: (1) the release of adsorbed water, crystal water and the attached acetone molecules (up to $\left.180^{\circ} \mathrm{C}\right)$; (2) the dehydroxylation of the metal-hydroxide layer $\left(180-300^{\circ} \mathrm{C}\right)$, with endothermic peaks at $230{ }^{\circ} \mathrm{C}$ in the DTA curve; (3) the decomposition of the guest anion $\left(300-500{ }^{\circ} \mathrm{C}\right)$, with endothermic peaks between 330 and $400{ }^{\circ} \mathrm{C}$ in the DTA curve. A partial overlap is observed between the second and third mass loss as usual. Such steps can estimate the organic content and resulting the chemical formulae of the D-LDH (h) (Table 1 ). The residual mass at $700{ }^{\circ} \mathrm{C}$ is consider as $" \mathrm{Zn}_{2} \mathrm{AlO}_{7 / 2}$ ", which links the molecular weight for D-LDH (h) to its total weight loss. Also, the water content was estimated by the loss percentage. According to the setting in terms of interleaved species, $\left(D^{-}\right)_{1-x}\left(C_{3}{ }^{2-}\right)_{x / 2} \mathrm{nH}_{2} \mathrm{O}$, the relative amount of each contribution is calculated against the LDH framework. The thermal stability is expressed by DTA and the temperatures at a determined mass loss (e.g., $50 \%$, at $\mathrm{T}_{50 \%}$ ). Interestingly, $\mathrm{T}_{50 \%}$ gradually increases from 305 to $363{ }^{\circ} \mathrm{C}$ according to the AMO time. Such the $T_{50 \%}$ difference of ca. $60^{\circ} \mathrm{C}$ is significant. Indeed, the D-LDH (h) shows high DTA exothermic peaks in the range from $333{ }^{\circ} \mathrm{C}$ for $\mathrm{D}-\mathrm{LDH}(\mathrm{Oh})$ to $397{ }^{\circ} \mathrm{C}$ for $\mathrm{D}$-LDH (6h). All these results suggest that the modification by acetone solvent improves the thermal stability of the guest species due to the interaction between acetone and D-LDH, but there is no obvious correlation between the thermal stability and the used AMO time.

Besides, the influence of solvent modification on the radical scavenging activity of DBHP molecule was examined using the DPPH• radical as the probe to examine the antioxidant ability of active compounds. ${ }^{23}$ Fig 4 a displays the UV-vis spectra of the DPPH $\bullet$ radical ethanol solution with a strong absorption band at $517 \mathrm{~nm}$, which exhibits dark purple. After being captured by the antioxidants, the intensity of this absorption peak decreases because of the formation of stable radical free compound, and then the colour of the ethanol solution turns pale yellow or colorless. ${ }^{24}$ Therefore, the radical-scavenging reaction can be used to monitor the change of this typical absorption band. Because of the co-intercalation of $\mathrm{D}^{-}$and $\mathrm{CO}_{3}{ }^{2-}$ during the AMO process, the amount of DBHP in DPPH• ethanol solution is different. Fig. 4b depicts the radical-scavenging activity (RCA) of D-LDH (h) according to the content of DBHP. It seems that the radical-scavenging activity tends to decrease with an increase in the content 
of interleaved DBHP molecules. This may be interpreted by the fact that more DBHP ions between layers lead to tighter arrangement. That maybe influence the contact between DBHP molecules and the surrounded DPPH •, which can reduce the radical scavenging activity.
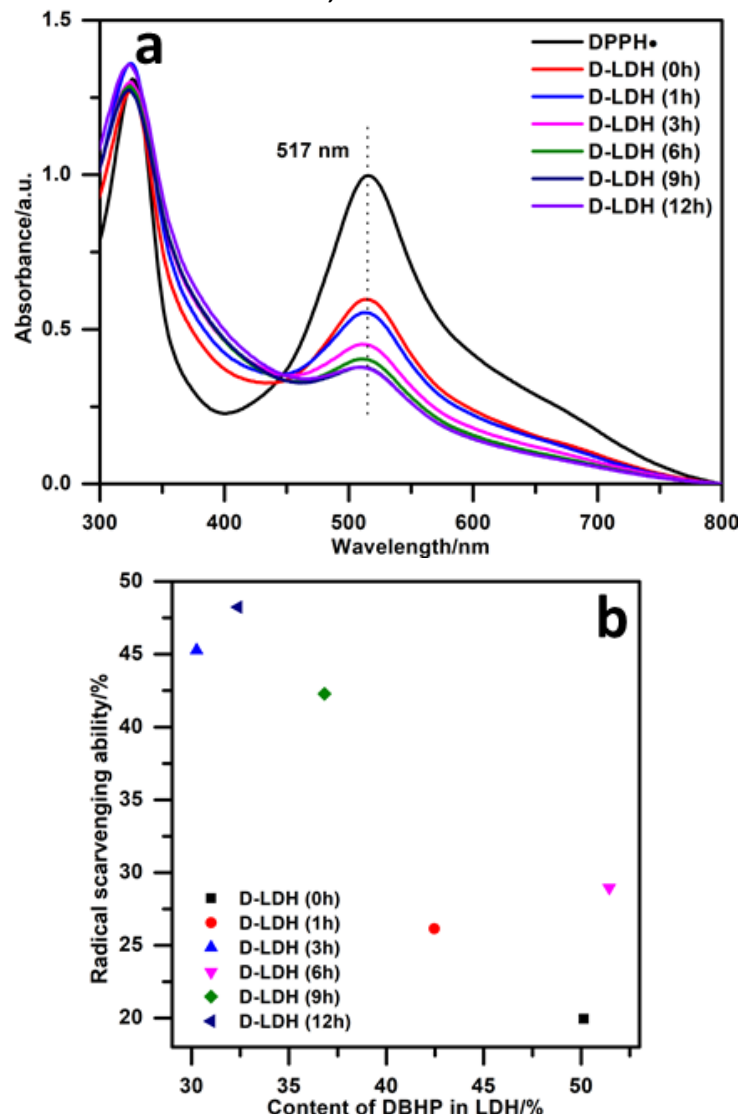

Fig. 4 (a) UV-vis spectra and (b) radical-scavenging activity of DBHP in D-LDH (h).

PP/D-LDH (h) composites

Fig. 5 displays the XRD patterns of PP/D-LDH (h) composites. In the low $2 \theta$ angular domain (1.5-10 $)$, when the D-LDH (h) are dispersed into PP, the corresponding XRD patterns for PP/D-LDH (h) composites show some characteristic (0Ol) reflections, especially for the composites PP/D-LDH (9h) and (12h). Compared with the pristine D-LDH (h) samples (Fig. 1a), the positions of the reflection (003) in PP/D-LDH (h) composites are slightly shifted to lower $2 \theta$ angles, corresponding to a basal spacing distance between 2.65 and $2.86 \mathrm{~nm}$. Such a lamellar expansion may indicate that some PP chains possibly diffuse into the LDH gap and then produce partially an intercalated polymer composite structure. ${ }^{25}$ At the larger angular domain, as identified in Fig. 5b, X-ray patterns represent the characteristic Bragg reflections of $\alpha$-form PP crystal, assigned to the planes of (110), (040), (130), (111) and (131)/(041), and the diffraction peaks are in the same positions for all the PP composites. However, the (300) diffraction peak of the $\beta$-form PP is observed in PP/D-LDH (1h) and barely visible in PP/D-LDH (9h) and (12h). ${ }^{26}$ The observation of $\beta$-PP illustrates that the incorporation of LDHs affects the crystallized domains of PP but such effect seems to be erratic and not correlated strictly to AMO time. 

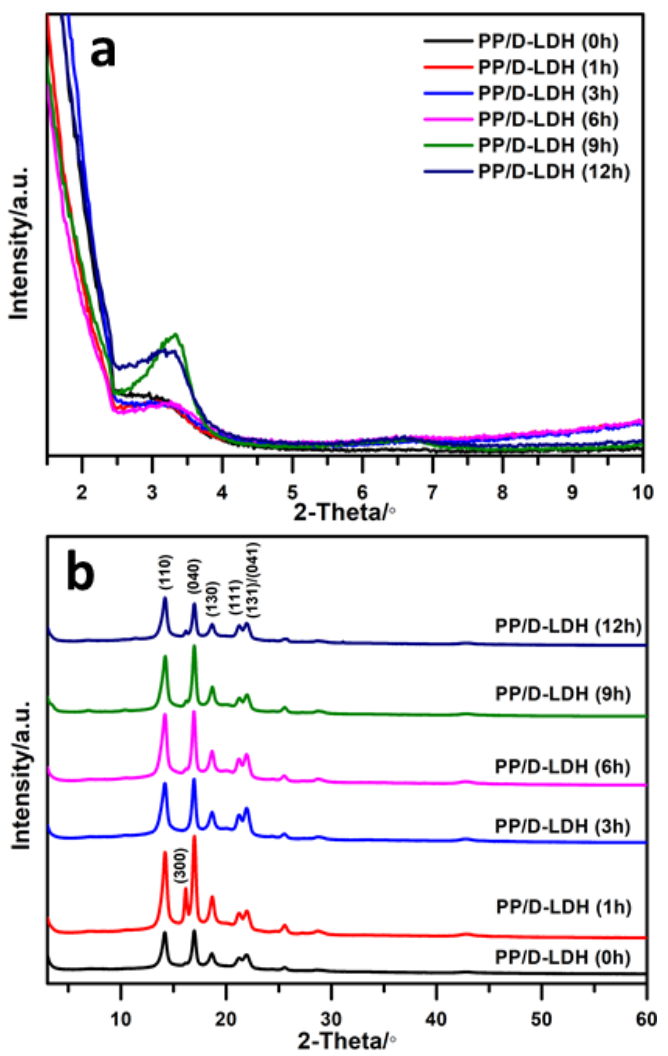

Fig. 5 XRD patterns of PP/D-LDH (h) composites in the range (a) $1.5-10^{\circ} / 2 \theta$ and (b) $3-60^{\circ} / 2 \theta$.

Fig. 6 shows SAXS results of PP and PP/D-LDH(h) for examination of the structural long-range correlation possibly present in the composites composed of D-LDH (h) fillers in PP. In comparison with PP (free of filler), the composites exhibit a stronger scattering intensity, evidently due to the presence of LDH platelets (electron contrast). For all the PP/D-LDH (h) samples, two peaks are observed at the diffusion value $q$ of 0.037 and $0.077 \AA^{-1}$, corresponding to the interlamellar distances of 2.7 and $1.3 \mathrm{~nm}$, respectively, and related to the (003) and (006) Bragg reflections of D-LDH (h) as discussed in XRD analysis (Fig. 1). Moreover, there is the third hump at lower q value, involving the size of the stacked particles agglomerates. For PP/D-LDH (Oh), a layer hump is visible at $q \sim 0.008 \AA^{-1}$, which corresponds to a size correlation of $12.5 \mathrm{~nm}$. This would have been located at $2 \theta=0.7^{\circ}$ therefore it is not observed through conventional X-Ray diffraction since it is in the steep rise of the direct diffraction beam. By increasing AMO time from 1 to $6 \mathrm{~h}$, the previous hump disappears and a slight change of slope is barely visualized at ca. $q=0.006 \AA-1$. This may be interpreted by a change of region from fractal to Porod domain. The absence of pronounced long-range correlation is in agreement with the de-agglomeration as observed when applying AMO method.14 At $q<0.004 \AA-1$, the slope becomes constant and similar for all PP/D-LDH (h) samples, suggesting the outside-surface agglomerates interacting with PP chains. A linear correlation fit of R2 $=0.99$ leads to a slope of about -1.7 , consistent with a scattering from sheet-like two-dimensional platelets of D-LDH (h), this almost on the entire q decade from $10^{-2} \AA^{-1}$ down to $10^{-3} \AA^{-1}$. $^{27}$ 


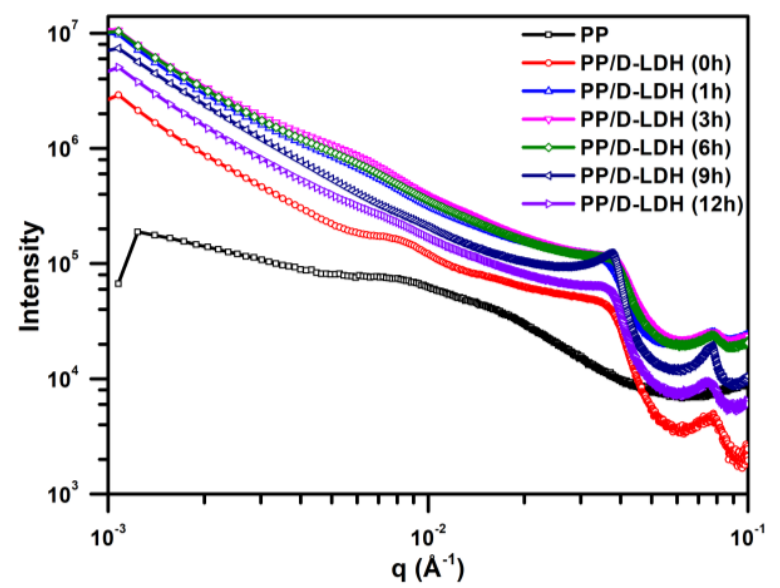

Fig. 6 SAXS data for PP/D-LDH (h) composites.
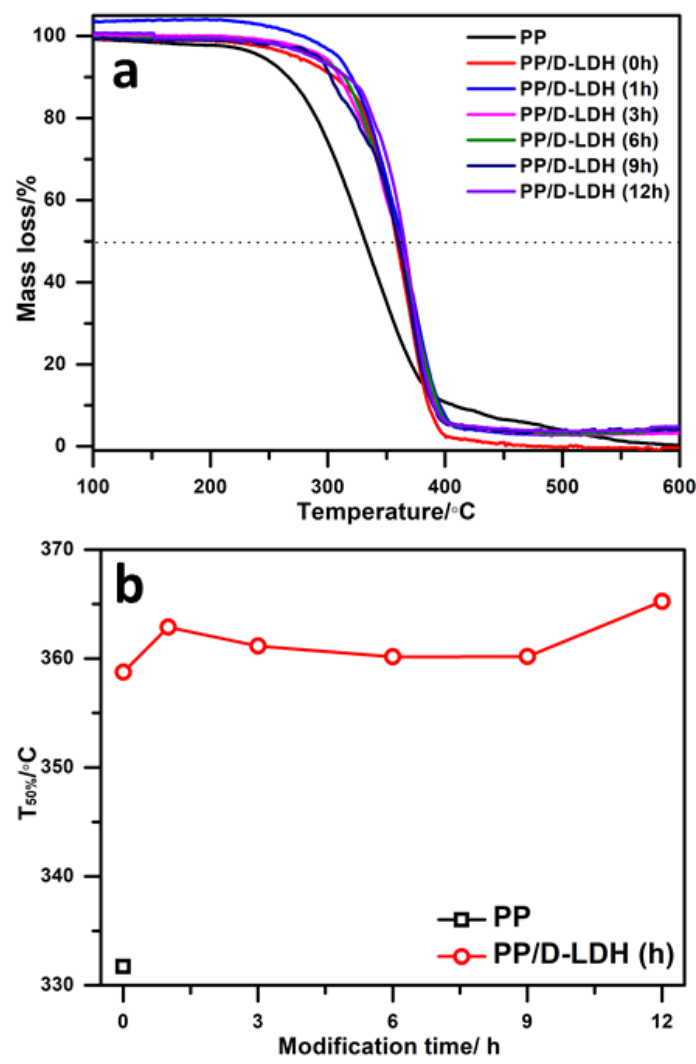

Fig. 7 (a) TGA curves and (b) $T_{50 \%}$ of PP/D-LDH (h) composites.

The thermal stability of the PP composites was investigated by thermogravimetric analysis (TGA) and FT-IR spectrometer. Fig. 7a shows the mass loss curves of PP and PP/D-LDH (h) composites. For all the composites, the major mass loss occurs between 250 and $400{ }^{\circ} \mathrm{C}$ with the similar trend. The $50 \%$ mass loss temperature (T50\%) is considered for comparison (Fig. 7b), T50\% values in presence of the hybrid LDH filler range between 358 and $365{ }^{\circ} \mathrm{C}$ are significantly higher than that of $331{ }^{\circ} \mathrm{C}$ for PP without filler. The difference of T50\% among the composites is small. As a result, with the addition of D-LDH (h), the thermal stability of the PP composites is obviously improved. However, the AMO time has little influence on the thermal stability of the PP/D-LDH (h) composites.

Also, Fig. 8a displays the FT-IR spectra for thermal-aging of PP film at $150{ }^{\circ} \mathrm{C}$ as a function of aging time. The PP film is totally broken after the thermal aging time of $160 \mathrm{~min}$, indicating that PP has the 
weak thermal stability. As the aging time increases, the intensity and the area of carbonyl absorption band of PP significantly increase, accompanying with discolor and distortion in appearance of the film. Here, the integrated area of carbonyl band quantifies the thermal oxidative degradation degree of PP/D-LDH (h) composites. Fig. 8b shows the integrated area of PP/D-LDH (h) composites after 40 h. Three time scales are observed during the thermal-oxidative degradation: (1) PP/D-LDH (Oh) has relatively rapid thermal aging and completely was broken down before $60 \mathrm{~h}$; (2) PP/D-LDH (1h), (3h) and (12h) present certain anti-aging stability until $100 \mathrm{~h}$; (3) PP/D-LDH (6h) and (9h) films exhibit an efficient stability against thermal aging, and their appearance remains intact even after $100 \mathrm{~h}$. Based on these observations, PP/D-LDH (Oh) composite exhibits weak ability against thermal aging, while the other D-LDH (h) samples show significantly enhanced capacity against the thermal aging to promote PP integrity. The small addition of D-LDH into PP polymer is somehow levelling off the difference in DBHP content between each filler, thus also for the series of PP/D-LDH composites. With the increase of AMO time, the anti-aging performance is gradually improved as the AMO time is about 6 to $9 \mathrm{~h}$ and then is reduced.
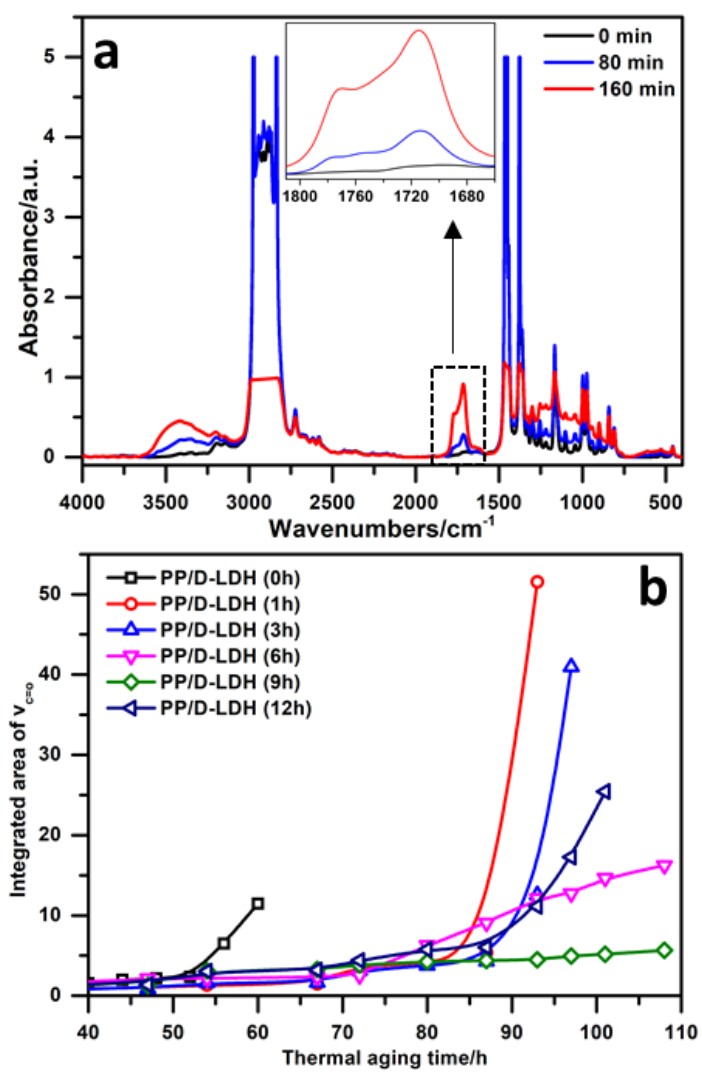

Fig. 8 (a) FT-IR spectra of PP during thermal aging; (b) The integrated area of carbonyl band of PP/D-LDH (h) composites between $1810-1660 \mathrm{~cm}^{-1}$.

To deeper investigate the migration properties of DBHP in PP/D-LDH (h) composites, UV-vis analysis was performed. DBHP molecule represents a typical absorption peak at $284 \mathrm{~nm}$ in UV-vis spectrum, attributed to the $\pi-\pi^{*}$ state. Fig. 9a presents the standard curve of UV absorption and DBHP concentration in ethanol solution with a good linear correlation ( $R 2=0.997)$ between the absorbance (A) and the concentration (C). Therefore, the intensity of the DBHP characteristic peak is used to monitor DBHP content as released from PP/D-LDH (h) composite. Fig. 9b further shows the release quantity versus time for PP/D-LDH (h) composites adopting 4 wt\% loading of D-LDH(h) in the composite. The migration of DBHP molecules increases rapidly in the first $12 \mathrm{~h}$, corresponding to the well-known "burst release" phenomenon. The release of DBHP molecules in the solution reaches the maximum value and eventually tends to the constant. Table 2 summarizes the migration amount of DBHP out of PP and the percent of initial DBHP in PP/D-LDH composites. For the series, the 
experimental results exhibit the amount of DBHP molecules migrated out of PP films in the range between 20.1 and $43.8 \mathrm{mg}$, corresponding to an ingress ratio ranging from 12.2 to $40.8 \%$, of the DBHP molecules initially interleaved. Compared with other PP/D-LDH film, PP/D-LDH (Oh) and (6h) represent the lowest migration in the series, corresponding to the D-LDH (h) with higher DBHP content. PP/D-LDH (9h) and (12h) samples show higher migration rates, suggesting that the lower content of DBHP and the longer modification time of D-LDH is not conducive to the immobilization of LDH particles in PP. Interestingly, PP/D-LDH (1h) composite exhibits migration rate similar to PP/DLDH (3h) composites, while the content of DBHP is really different. Both the amount of DBHP and the modification time affect the migration of PP/D-LDH (h) composites.
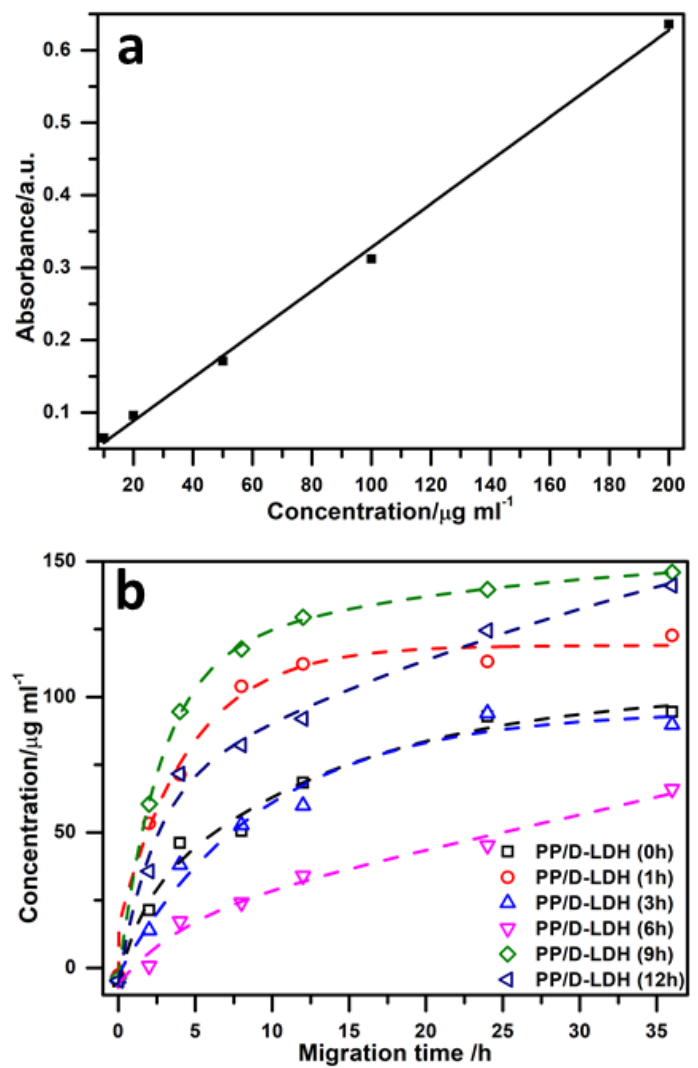

Fig. 9 (a) Standard curve of absorbance and concentration of DBHP in ethanol solution; (b) The migration curves of PP/D-LDH (h) composites.

Table 2 Migration results of PP/D-LDH (h).

\begin{tabular}{llll}
\hline Samples & $\begin{array}{l}\text { D in PP } \\
(\mathrm{mg})\end{array}$ & $\begin{array}{l}\text { D in ethanol } \\
\text { solution }(\mathrm{mg})\end{array}$ & $\begin{array}{l}\text { Release } \\
\text { of } \mathrm{D}(\%)\end{array}$ \\
\hline PP/D-LDH (Oh) & 160.4 & 28.3 & 17.6 \\
PP/D-LDH (1h) & 135.9 & 36.8 & 27.1 \\
PP/D-LDH (3h) & 96.8 & 26.9 & 27.8 \\
PP/D-LDH (6h) & 164.6 & 20.1 & 12.2 \\
PP/D-LDH (9h) & 117.9 & 43.8 & 37.1 \\
PP/D-LDH (12h) & 103.7 & 42.3 & 40.8
\end{tabular}

\section{Conclusions}


In this work, a series of AMO D-LDH (h) antioxidants with different acetone modification times were prepared by an AMO method. The AMO method does not affect the layer structure of D-LDH but causes a contamination by $\mathrm{CO}_{2}$. The co-intercalation of $\mathrm{D}^{-}$and $\mathrm{CO}_{3}{ }^{2-}$ in $\mathrm{D}-\mathrm{LDH}(\mathrm{h})$ change the amount of DBHP in the interlayer and then influence the crystallinity, thermal stability and the radical-scavenging activity of D-LDH (h). For PP/D-LDH (h) composites, longer AMO modification time or greater amount of DBHP molecules in D-LDH is found to be not necessarily conducive to obtain the best performance for PP composite in terms of stability. In comparison, the PP/D-LDH (9h) film exhibits a good stability against thermal aging while the PP/D-LDH (6h) film represents the lowest migration in all composites. The AMO modification of D-LDH affects the content of DBHP and the interaction between LDH filler and PP chains, noticeably evidenced by the fact that an optimized modification improves the anti-aging performance of D-LDH for PP, as well as impeding the migration of DBHP molecules out of PP films.

\section{Experimental}

\section{Chemicals.}

Magnesium nitrate $\left(\mathrm{Mg}\left(\mathrm{NO}_{3}\right)_{2} \cdot 6 \mathrm{H}_{2} \mathrm{O}\right)$, aluminum nitrate $\left(\mathrm{Al}\left(\mathrm{NO}_{3}\right)_{3} \cdot 9 \mathrm{H}_{2} \mathrm{O}\right)$, sodium hydroxide, acetone, methanol and xylene were reagent grade and procured from Beijing Tongguang Chemical Co. Ltd. The 2,2-diphenyl-1picrylhydrazyl (DPPH•) was purchased from Shanghai Macklin Chemical Co. Ltd. Polypropylene (PP1300) was supplied by Sinopec Beijing Yanshan Company. All the chemicals were used as received.

\section{Synthesis of D-LDH (h).}

The conventional D-LDH was prepared using a coprecipitation method. Briefly, the salt solution (50 $\mathrm{mL})$ of $0.4 \mathrm{M}$ $\mathrm{Mg}\left(\mathrm{NO}_{3}\right)_{2} \cdot 6 \mathrm{H}_{2} \mathrm{O}$ and $0.2 \mathrm{M} \mathrm{Al}\left(\mathrm{NO}_{3}\right)_{3} \cdot 9 \mathrm{H}_{2} \mathrm{O}$ was added drop-wise into a $0.4 \mathrm{M} \mathrm{DBHP}$ aqueous solution (50 mL, with $0.02 \mathrm{~mol} \mathrm{NaOH}$ previously) under rapid stirring and nitrogen bubbling. The $\mathrm{pH}$ value was kept at 9 10 using a 2.0 $\mathrm{M} \mathrm{NaOH}$ solution. This addition process was controlled in $30 \mathrm{~min}$. After aging for $12 \mathrm{~h}$ at room temperature, the suspension was centrifugated and washed by distilled water until the $\mathrm{pH}$ was close to 7 . In the AMO method, the wet cake D-LDH was washed with acetone thoroughly, named as D-LDH (Oh). The D-LDH (Oh) washed with acetone was then dispersed in $150 \mathrm{~mL}$ of acetone and stirred at room temperature during different times ( 1 to $12 \mathrm{~h}$ ). The AMO D-LDHs were obtained by filtration and a part of the slurries was dried overnight for structural analysis. As a function of the time of stirring into acetone, the AMO D-LDH samples were named as D-LDH $(\mathrm{h})$, with modification time from 0 to $12 \mathrm{~h}$.

\section{Preparation of PP/D-LDH (h) composites.}

The AMO D-LDH (h) slurries were used directly to prepare PP/D-LDH (h) composites using a solvent mixing method. ${ }^{28}$ For example, $10 \mathrm{~g}$ of $\mathrm{PP}$, and $150 \mathrm{~mL}$ of xylene were transferred into a $500 \mathrm{~mL}$ round bottom flask in presence of D-LDH slurry. For the latter, the weight percentage added to PP was confirmed to be $4.0 \mathrm{wt} \%$, according to the solid content into LDH slurries. The mixture was stirred at $120^{\circ} \mathrm{C}$ for $12 \mathrm{~h}$ to dissolve PP and to yield highly dispersed LDH platelets. After this solvent mixing process, the hot xylene mixture was poured into 50 $\mathrm{mL}$ methanol for the extraction of a white precipitate. The PP/D-LDH (h) composites were collected by filtration and dried in the oven to remove the residual xylene. The PP/D-LDH (h) films were prepared by hot-press between two Teflon sheets at $170{ }^{\circ} \mathrm{C}$ with the thickness of $0.1 \mathrm{~mm}$.

\section{Characterization techniques.}

The X-ray diffraction (XRD) patterns were recorded on the Bruker D8 Advance XRD system at $40 \mathrm{kV}$ and $40 \mathrm{~mA}$ with a $\mathrm{Cu} \mathrm{K \alpha}$ radiation $\left(\lambda=0.154 \mathrm{~nm}\right.$ ) in the range of $2 \theta=2-70^{\circ}$. Small Angle X-ray Scattering (SAXS) were examined on an Empyrean Panalytical equipment with ScatterX78 using a $\theta / \theta$ goniometer and $\mathrm{Cu}$ anode $(40 \mathrm{kV}$ and $40 \mathrm{~mA}$ ). The curves were collected in a continuous scan mode with the background removed. Fourier transform infrared spectra (FT-IR) were performed on Bruker Vector 22 infrared spectrophotometer in the range of $4000-400 \mathrm{~cm}^{-1}$, with 32 scans and a resolution of $2 \mathrm{~cm}^{-1}$. The powder LDHs and PP/LDH composites were tested using thin $\mathrm{KBr}$ pellets and films respectively. SEM analyses were obtained using a Zeiss scanning electron microscope. The powder LDHs were dispersed in ethanol for sonication and then casted on a silicon wafer. Thermogravimetric Analyse (TGA) were monitored on PCT-IA instrument in the range of $25-700{ }^{\circ} \mathrm{C}$ with a heating rate of $5{ }^{\circ} \mathrm{C} / \mathrm{min}$. The Ultraviolet-visible spectra (UV-vis) from 200 to $800 \mathrm{~nm}$ were measured by using a Shimadzu UV-2501PC spectrophotometer. 


\section{Antioxidant activity of D-LDH (h).}

To evaluate the antioxidant activities of AMO D-LDH $(\mathrm{h})$, the radical scavenging abilities were determined according to the DPPH• assay in an ethanol solution. ${ }^{29}$ The powder D-LDH (h) samples were added into $100 \mathrm{~mL}$ $\mathrm{DPPH} \bullet$ ethanol solution $(100 \mu \mathrm{M})$ and the reaction was carried out at room temperature for $30 \mathrm{~min}$. The decrease of $\mathrm{DPPH} \bullet$ concentration was measured by recorded the UV-vis absorbance at $517 \mathrm{~nm}$. The radical-scavenging ability (RSA) of each D-LDH (h) sample was calculated by the following equation: RSA $(\%)=\left(A_{D P P H}-A_{L D H}\right) / A_{D P P H} \times 100$ ( $A_{\mathrm{DPPH}}$ : absorbance of DPPH $\bullet$ at $30 \mathrm{~min} ; \mathrm{A}_{\mathrm{LDH}}$ : absorbance of DPPH・ and D-LDH (h) at $30 \mathrm{~min}$ ).

\section{Thermal aging of PP/D-LDH (h) composites.}

The thermal oxidative degradation of PP/D-LDH $(\mathrm{h})$ composites were investigated by accelerated thermal-aging test. The composite films were tailored to the size of $20 \times 20 \mathrm{~mm}$ and placed at $150{ }^{\circ} \mathrm{C}$. The FT-IR spectra of PP/D$\mathrm{LDH}(\mathrm{h})$ composite films were monitored every 2 hours and the change of carbonyl group was defined to evaluate the rate of the thermal oxidative degradation. ${ }^{30}$

\section{Migration of PP/D-LDH (h) composites}

The migration test of antioxidant from PP was as follows. Based on the linear relationship between the UV-vis absorbance $(284 \mathrm{~nm})$ and the concentration of DBHP species in ethanol solution, the content of antioxidant DBHP in solution was measured. In brief, $8.0 \mathrm{~g} \mathrm{PP} / \mathrm{D}-\mathrm{LDH}$ (h) film and $300 \mathrm{~mL}$ of ethanol were transferred into a conical flask and then placed in an oven at $60^{\circ} \mathrm{C}$. The amount of DBHP molecules released out of PP was monitored at different immersion times, through the UV-vis spectrometer.

\section{Conflicts of interest}

There are no conflicts to declare.

\section{Acknowledgements}

This work is supported by the National Natural Science Foundation of China, the Fundamental Research Funds for the Central Universities (JD1716, 12060093063), National Basic Research Program of China (973 program, 2014CB932104), and the Program for Changjiang Scholars and Innovative Research Team in University (No. IRT1205). Also, Miss Qian Zhang gratefully acknowledges the financial support from China Scholarship Council (File No. 201906880038).

\section{Notes and references}

1 D. Dhamodharan, T. Senthilc, M. B. Kale, S. Mubarak, N. Divakaran, L. Wu and J. Wang, Compos. Part A, 2019, 124, 105480.

2 J. He, J. Chen, L. Shi, Q. Li, W. Lu, S. Qu, W. Qiu and G. Zhou, Carbon, 2019, 147, 236-241.

3 X. Chen, Y. Huang and K. Zhang, J. Colloid Interf. Sci., 2018, 513, 788-796.

4 Q. Wang and D. O'Hare, Chem. Rev., 2012, 112, 4124-4155.

5 Z. Gu, J. J. Atherton and Z. P. Xu, Chem. Commun., 2015, 51, 3024-3036.

6 M. Kotal and A. K. Bhowmick, Prog. Polym. Sci., 2015, 51, 127-187.

7 H. Zhong, C. Campos-Roldán, Y. Zhao, S. Zhang, Y. Feng and N. Alonso-Vante, Catalysts, 2018, 8, 559.

8 C. Li, M. Wei, D. G. Evans and X. Duan, Small, 2014, 10, 4469-4486.

9 Y. Hou, M. R. Lohe, J. Zhang, S. Liu, X. Zhuang and X. Feng, Energ. Environ. Sci., 2016, 9, 478-483.

10 X. Mei, W. Wang, L. Yan, T. Hu, R. Liang, D. Yan, M. Wei, D. G. Evans and X. Duan, Biomaterials, 2018, 165, 14-24.

11 C. Tan, X. Cao, X. Wu, Q. He, J. Yang, X. Zhang, J. Chen, W. Zhao, S. Han, G. H. Nam, M. Sindoro and H. Zhang, Chem. Rev., 2017, 117, 6225-6331.

12 S. Mallakpour and M. Hatami, Eur. Polym. J., 2017, 90, 273-300.

13 Q. Wang and D. O'Hare, Chem. Commun., 2013, 49, 6301-6303.

14 C. Chen, M. Yang, Q. Wang, J. C. Buffet and D. O'Hare, J. Mater. Chem. A, 2014, 2, 15102-15110.

15 Y. Gao, Y. Zhang, G. R. Williams, D. O'Hare and Q. Wang, Sci. Rep., 2016, 6, 35502.

16 Y. Feng, Y. Jiang, Q. Huang, S. Chen, F. Zhang, P. Tang and D. Li, Ind. Eng. Chem. Res., 2014, 53, 2287-2292.

17 Q. Zhang, F. Leroux, P. Tang, D. Li and Y. Feng, Polym. Degrad. Stab., 2018, 154, 55-61.

18 Q. Zhang, Q. Jiao, F. Leroux, P. Tang, D. Li and Y. Feng, New J. Chem., 2017, 41, 2364-2371.

19 R. Liu, Y. Wang, D. Liu, Y. Zou and S. Wang, Adv. Mater., 2017, 29, 1701546.

20 L. Qiu, Y. Gao, P. Lu, D. O'Hare and Q. Wang, J. Appl. Polym. Sci., 2018, 46204.

21 Q. Wang, X. Zhang, C. J. Wang, J. Zhu, Z. Guo and D. O'Hare, J. Mater. Chem. A, 2012, 22, 19113-19121.

22 Q. Zhou, V. Verney, S. Commereuc, I. J. Chin and F. Leroux, J. Colloid Interface Sci., 2010, 349, 127-133. 
23 S. B. Kedare and R. P. Singh, J. Food Sci. Technol., 2011, 48, 412-422.

24 Y. Deligiannakis, G. A. Sotiriou and S. E. Pratsinis, ACS Appl. Mater. Interfaces, 2012, 4, 6609-6617.

25 G. Totaro, L. Sisti, A. Celli, I. Aloisio, D. Di Gioia, A. A. Marek, V. Verney and F. Leroux, Dalton Trans., 2018, 47, 3155-3165.

26 G. G. Wu, C. Ding, W. B. Chen, Y. Zhang, W. Yang and M. B. Yang, Polymer, 2019, 175, 177-185.

27 D. W. Schaefer and R. S. Justice, Macromolecules, 2007, 40, 8501-8517.

28 Q. Wang, X. Zhang, J. Zhu, Z. Guo and D. O'Hare, Chem. Commun., 2012, 48, 7450-7452.

29 K. H. Musa, A. Abdullah and A. Al-Haiqi, Food Chem., 2016, 194, 705-711.

30. Q. Zhang, Y. Guo, A. A. Marek, V. Verney, F. Leroux, P. Tang, D. Li and Y. Feng, Inorg. Chem. Front., 2019, 6, 2539-2549. 